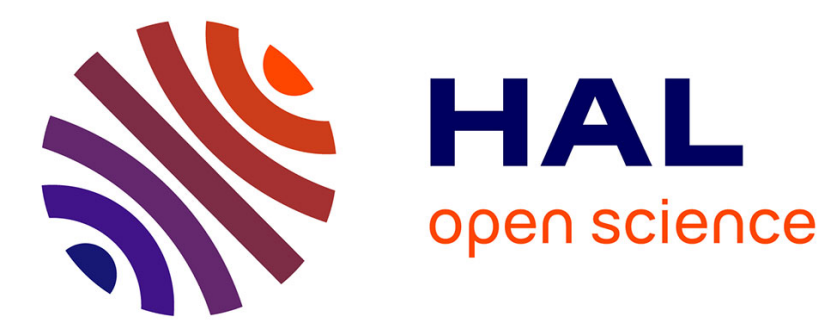

\title{
Satisfaction-based Radio Access Technology Selection in Heterogeneous Wireless Networks
}

\author{
Melhem El Helou, Samer Lahoud, Marc Ibrahim, Kinda Khawam
}

\section{To cite this version:}

Melhem El Helou, Samer Lahoud, Marc Ibrahim, Kinda Khawam. Satisfaction-based Radio Access Technology Selection in Heterogeneous Wireless Networks. Wireless Days (WD), 2013 IFIP, Nov 2013, Spain. 4 p., 10.1109/WD.2013.6686461 . hal-01018234

\section{HAL Id: hal-01018234 https://hal.science/hal-01018234}

Submitted on 3 Jul 2014

HAL is a multi-disciplinary open access archive for the deposit and dissemination of scientific research documents, whether they are published or not. The documents may come from teaching and research institutions in France or abroad, or from public or private research centers.
L'archive ouverte pluridisciplinaire HAL, est destinée au dépôt et à la diffusion de documents scientifiques de niveau recherche, publiés ou non, émanant des établissements d'enseignement et de recherche français ou étrangers, des laboratoires publics ou privés. 


\title{
Satisfaction-based Radio Access Technology Selection in Heterogeneous Wireless Networks
}

\author{
Melhem El Helou*†, Samer Lahoud ${ }^{\dagger}$, Marc Ibrahim* and Kinda Khawam ${ }^{\ddagger}$ \\ * Saint-Joseph University, ESIB, Campus des Sciences et Technologies, Mar Roukoz, Lebanon \\ ${ }^{\dagger}$ University of Rennes 1, IRISA, Campus de Beaulieu, 35042 Rennes, France \\ ‡University of Versailles, PRISM, 45 Avenue des Etats-Unis, 78035 Versailles, France
}

\begin{abstract}
In this paper, a hybrid approach for Radio Access Technology (RAT) selection in heterogeneous wireless networks is proposed. This decision framework dynamically integrates operator objectives and user preferences, with a relatively reduced network complexity, signaling and processing load. By broadcasting cost and QoS parameters, the network assists mobile users in their decisions. Focusing on the user side, we present a satisfaction-based multi-criteria decision-making (MCDM) method. Based on their needs and preferences, individual users select their RAT avoiding inadequate decisions. Simulation results show that our MCDM method maximizes user utility and outperforms existing solutions.
\end{abstract}

Index Terms-Radio access technology selection, heterogeneous wireless networks, multi-criteria decision-making.

\section{INTRODUCTION}

Along with the rapid growth of mobile broadband traffic, efficient RAT selection techniques are increasingly required. In the recent few years, many network-centric approaches [1]-[3] have been studied. When network elements collect necessary measurements and information, they take selection decisions transparently to end-users in a way to meet operator objectives (e.g., enhance resource utilization, lower energy consumption). However, to reduce network complexity, signaling and processing load, mobile-terminal-centric approaches [3]-[6] have also gained in importance. Based on their individual needs and preferences, rational users select their RAT so as to selfishly maximize their utility. Yet, because mobiles have no information on the global network state (i.e., dynamic load conditions), mobile-terminal-centric methods are known for their potential inefficiency.

In this article, we propose a hybrid approach that combines benefits from both network-centric and mobile-terminalcentric methods. On the one side, by broadcasting appropriate cost and QoS parameters, the network tries to globally control users decision in a way to meet operator objectives. On the other side, based on their needs and preferences as well as on the signaled network information, individual users select their RAT so as to maximize their own utility.

We have previously introduced in [7] two tuning policies that dynamically derive network information as a function of the load conditions. In the present contribution, we focus on the user side and propose a satisfaction-based MultiCriteria Decision-Making (MCDM) method. In comparison with existing MCDM algorithms, namely the Simple Additive Weighting (SAW) [5], and the Technique for Order Preference by Similarity to Ideal Solution (TOPSIS) [6], our method avoids undersized and oversized decisions providing better performances and higher user satisfaction.

\section{HYBRID DECISION FRAMEWORK}

\section{A. Network information}

Network information is periodically sent to all mobile users using the logical communication channel (i.e., radio enabler) proposed by the IEEE standard 1900.4 [8]. When a new or a handover session arrives, the mobile decodes this decisional information, evaluates available alternatives, and selects the RAT that best suits it.

In this setting, we assume that the network information provides cost and some QoS parameters: they can be seen as incentives to join available RATs.

- Cost parameters: A volume-based fixed pricing strategy is proposed. Mobiles are then charged based on the amount of traffic they consume.

- QoS parameters: Throughputs that can be allocated to future arrivals are broadcasted:

- Mobiles are guaranteed an average minimum throughput, denoted by $d_{\text {min }}$.

- They also have priority to benefit from an average maximum throughput, denoted by $d_{\max }$.

However, since perceived throughputs highly depend on radio conditions (or equivalently on adopted modulation types and FEC coding rates), $d_{\min }$ and $d_{\max }$ are derived for the most robust modulation and coding scheme. Therefore, when evaluating available alternatives, mobiles should combine their individual radio conditions with the provided QoS parameters: for that they multiply $d_{\min }$ and $d_{\max }$ with a given modulation and coding gain, denoted by $g(M, C)$.

\section{B. RAT selection}

For alternative $a$, the network broadcasts the three parameters: $d_{\min }(a), d_{\max }(a)$, and $\operatorname{cost}(a)$. New arrivals then compute a utility function for each of the available alternatives, and select the one with the highest score. This utility, obtained after normalizing and weighting the decision criteria, depends on user radio conditions, needs and preferences (e.g., QoSmaximizing, cost-minimizing preferences) as well as on the cost and QoS parameters signaled by the network. The particularity of our RAT selection process resides in the normalization 
step that takes into account user traffic class and throughput demand, thus avoiding inadequate decisions.

\section{SATISFACTION-BASED DECISION METHOD}

\section{A. Normalization and Traffic classes}

In our work, we consider three traffic classes : inelastic, streaming, and elastic classes. For traffic class $c$ and alternative $a, \hat{d}_{\min }^{c}(a), \hat{d}_{\max }^{c}(a)$, and $\widehat{\operatorname{cost}}^{c}(a)$ are respectively the normalized values of $d_{\min }(a), d_{\max }(a)$, and $\operatorname{cost}(a)$. Before we give the normalizing functions for each traffic class, we note that $\hat{p}^{c}(a), p \in\left\{d_{\min }, d_{\max }\right.$, cost $\}$, can be viewed as the satisfaction of a class $c$ session with respect to criterion $p$ for alternative $a$ :

- Inelastic sessions $(c=I)$ : Since they require stringent and deterministic bandwidth guarantees, $d_{\max }$ should not have any impact on the final decision. Besides, the satisfaction with respect to $d_{\min }$ has a step shape: mobiles expect to be satisfied when $d_{\min }$ is greater or equal to their fixed throughput demand $R_{f}$. Otherwise, they are not satisfied.

$$
\hat{d}_{\text {min }}^{I}(a)= \begin{cases}0 & \text { if } d_{\min }(a) \cdot g(M, C)<R_{f} \\ 1 & \text { if } d_{\min }(a) \cdot g(M, C) \geq R_{f}\end{cases}
$$

- Streaming sessions $(c=S)$ : Since they are usually characterized by a minimum, an average and a maximum bandwidth requirement, their throughput satisfaction is modeled as an sigmoid function:

$$
\hat{d}^{\prime}(a)=1-\exp \left(\frac{-\alpha\left(\frac{d^{\prime}(a) \cdot g(M, C)}{R_{a v}}\right)^{2}}{\beta+\left(\frac{d^{\prime}(a) \cdot g(M, C)}{R_{a v}}\right)}\right)
$$

where $d^{\prime}=\left\{d_{\min }, d_{\max }\right\}$.

$R_{a v}$ represents session needs: an average throughput demand. $\alpha$ and $\beta$ are two positive constants that determine the shape of the sigmoid function.

- Elastic sessions $(c=E)$ : Since they adapt to resource availability (i.e., load conditions), requiring no QoS guarantees, $d_{\min }$ is completely ignored. Moreover, the satisfaction with respect to $d_{\max }$ has a concave shape: the satisfaction increases slowly as the throughput exceeds the comfort throughput demand $R_{c}$ of the user (i.e., the mean throughput beyond which, user satisfaction exceeds $63 \%$ of maximum satisfaction).

$$
\hat{d}_{\text {max }}^{E}(a)=1-\exp \left(-\frac{d_{\max }(a) \cdot g(M, C)}{R_{c}}\right)
$$

The monetary cost satisfaction is, however, modeled as a Zshaped function: the slope of the satisfaction curve increases rapidly with the cost.

$$
\left.\widehat{\operatorname{cost}}^{c}(a)=\exp \left(-\frac{\operatorname{cost}(a)^{2}}{\lambda^{c}}\right), c \in\{I, S, E\}\right)
$$

$\lambda^{c}$ represents the cost tolerance parameter: a positive constant that determines the shape of the Z-shaped function.

\section{B. User Profile and Utility Function}

The user profile defines the cost tolerance parameter and the weights that a given session will apply to normalized criteria. More precisely, the user profile is the set of vectors $\left(\lambda^{c}, w_{d_{\min }}^{c}, w_{d_{\max }}^{c}, w_{\text {cost }}^{c}\right), c \in\{I, S, E\}$, where $w_{p}^{c}$ is the weight of $\hat{p}^{c}, p \in\left\{d_{\min }, d_{\max }\right.$, cost $\}$. The utility function of a class $c$ session for alternative $a$ is defined by :

\section{$U^{c}(a)=w_{d_{\text {min }}}^{c} \cdot \hat{d}_{\text {min }}^{c}(a)+w_{d_{\max }}^{c} \cdot \hat{d}_{\max }^{c}(a)+w_{\text {cost }}^{c} \cdot \widehat{\operatorname{cost}}^{c}(a)$ \\ IV. Simulation PARAMETERS AND RESUlTS}

For illustration, three generic OFDM(A)-based RATs are considered; each is assumed to propose three different service classes, namely Premium, Regular and Economic. For the sake of simplicity, all mobiles are supposed to have the same modulation and coding scheme. Cost and QoS parameters, as perceived by mobile users, are depicted in Table I. They are supposed fixed and do not change as the RAT load changes (except when the RAT is no longer able to guarantee to future arrivals the initial QoS parameters).

\begin{tabular}{cccc}
\hline Service class & $d_{\min }(\mathbf{M b} / \mathbf{s})$ & $d_{\max }(\mathbf{M b} / \mathbf{s})$ & $\operatorname{cost}(\mathbf{u n i t} / \mathbf{k B})$ \\
\hline Premium & 1.5 & 2 & 6 \\
Regular & 1 & 1.5 & 4 \\
Economic & 0.5 & 1 & 2 \\
\hline \multicolumn{4}{c}{ TABLE I }
\end{tabular}

STATIC QOS AND COST PARAMETERS

We assume that RAT capacity is fixed to $35 \mathrm{Mb} / \mathrm{s}$. The radio resource is however divided into 700 resource units (RU). In the time domain, transmissions are further organized into radio frames of $10 \mathrm{~ms}$ length. At each scheduling epoch, resource units are allocated to individual users based on their priority and current needs (i.e., amount of traffic waiting for transmission). Before any scheduling is applied, the minimum guaranteed throughputs (the operator guaranteed commitments) are directly granted. Then, the Weighted Fair Queuing is adopted to share out the remaining resources; grants are however limited to $d_{\max }$. Session priorities are based on the cost they pay for one unit of traffic. The residual resources are afterwards equitably distributed (according to the Round Robin service discipline).

We further suppose that mobile users arrive sequentially. The total number of users is however limited to $N_{\text {total }}$; it sets the traffic load. Their sojourn time is considered to be much greater in comparison with the simulation time. Consequently, the network dynamics will progressively slow down until a pseudo-stationary regime is attained, where all measurements are performed. Results are validated through extensive simulations.

After they arrive, mobiles are uniformly associated with a user profile (cf. Table II). The needs of inelastic and streaming sessions are respectively expressed as fixed (i.e., $R_{f}$ ) and average long-term throughput (i.e., $R_{a v}$ ). We assume that the set of possible throughput demands is given by $D=\{0.5,1,1.5,2\} \mathrm{Mb} / \mathrm{s}$. Inelastic sessions generate packets according to a deterministic distribution, whereas streaming 
sessions generate packets according to a Poisson process. In our work, we fix delay constraints for the latter session types. A maximum delay requirement of $100 \mathrm{~ms}$ is fixed. Since resources are limited, some packets may miss their deadline; they will be dropped as they are no longer useful.

\begin{tabular}{cccccc}
\hline Profile No. & Traffic class & $\lambda$ & $w_{d_{\min }}$ & $w_{d_{\max }}$ & $w_{\text {cost }}$ \\
\hline 1 & Inelastic & 60 & 0.7 & 0 & 0.3 \\
2 & Streaming & 60 & $14 / 30$ & $7 / 30$ & 0.3 \\
3 & Elastic & 60 & 0 & 0.7 & 0.3 \\
& Inelastic & 25 & 0.3 & 0 & 0.7 \\
4 & Streaming & 25 & 0.2 & 0.1 & 0.7 \\
5 & Elastic & 25 & 0 & 0.3 & 0.7 \\
6 & \multicolumn{5}{c}{ TABLE II } \\
\hline \multicolumn{5}{c}{ DETAILED USER PROFILES }
\end{tabular}

Furthermore, the needs of elastic sessions are expressed as comfort throughput (i.e., $R_{c}$ ). We suppose that the set of possible comfort throughputs is given by $C=\{0.75,1.25\}$ $\mathrm{Mb} / \mathrm{s}$. While inelastic and streaming sessions uniformly choose one of the possible throughput demands (regardless of the user cost tolerance parameter), we assume in the following that the comfort throughput of elastic sessions is related to the user willingness to pay and thus imposed by the user profile.

The proposed Satisfaction-Based (SB) MCDM method is compared with the well-known SAW and TOPSIS schemes. Because they ignore user needs, SAW and TOPSIS often lead to undersized and oversized decisions. Their decisions exclusively depend on user preferences (i.e., weights of the decision criteria) as well as on the available alternatives, without aligning with user throughput demands. So as to make the comparison more fair, enhanced SAW and TOPSIS are used: they only explore feasible alternatives. When their throughput demand is greater than the provided $d_{\max }$, the alternative opted for is considered to be infeasible and thus rejected. This will prevent SAW and TOPSIS from making some undersized decisions. However, as shown in the following, our proposed method continues to outperform them.

\section{A. Performance Evaluation}

To better analyze performance results, inelastic, streaming and elastic sessions are individually addressed.

1) Inelastic sessions: When SB is used, selection decisions are optimized to meet the exact session requirements, regardless of the user cost tolerance (willingness to pay for better performances or to save up money). However, because their normalization process ignores traffic class and needs, enhanced SAW and TOPSIS still lead to undersized and oversized decisions.

When users are ready to pay for better performances (i.e., users with profile no. 1), SAW and TOPSIS always single out to the Premium service class. Intuitively and since inelastic session needs are constantly fixed, this decision is oversized for 0.5 and $1 \mathrm{Mb} / \mathrm{s}$ sessions. As SB respectively opts for the Economic and the Regular service classes, QoS requirements are always perfectly satisfied, while cost is reduced.
Also, when users seek to save up money (i.e., users with profile no. 4), enhanced SAW and TOPSIS lead to the Economic service class for $1 \mathrm{Mb} / \mathrm{s}$ sessions and to the Regular one for $1.5 \mathrm{Mb} / \mathrm{s}$ sessions (undersized decisions). When the RAT is highly loaded, fixed QoS requirements are not satisfied, thus dramatically degrading session performances.

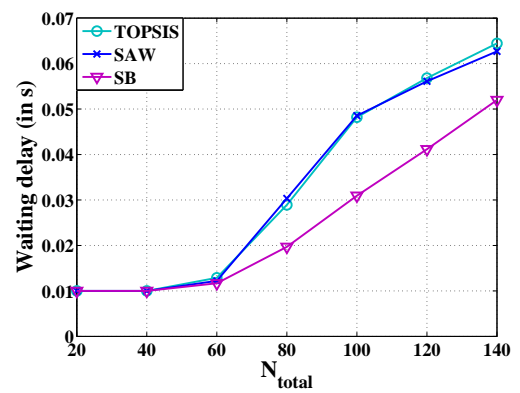

Fig. 1. Mean packet delay for inelastic sessions

Figures 1 and 2 respectively show the mean waiting delay and the packet drop probability as a function of the total number of arrivals. Since it avoids undersized decisions, SB provides a shorter delay, a lower drop probability and subsequently a better overall QoS level.

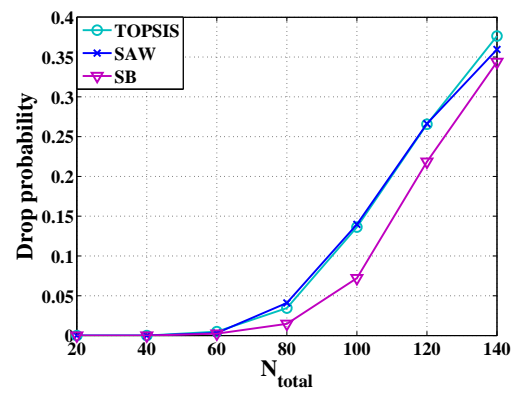

Fig. 2. Packet drop probability for inelastic sessions

We depict in Fig. 3(a) the average user satisfaction. We notice that, at low traffic load, enhanced SAW and TOPSIS provide higher satisfaction: First, undersized decisions are able to fulfill strict QoS requirements, while charging mobile users less. Second, although oversized decisions decrease user satisfaction, the reduction is not significant enough to offset the impact of undersized decisions. In other words, at low traffic load, undersized decisions considerably increase user satisfaction because the corresponding users seek to save up money; their QoS needs are perfectly met, while paying less. However, oversized decisions do not significantly decrease user satisfaction because users in question are originally ready to pay. We further note that, when traffic load is moderate, SB brings the largest satisfaction since it always meets the strict QoS requirements. Actually, under SAW and TOPSIS, undersized decisions are no more able to meet the QoS needs when traffic load is relatively high.

2) Streaming sessions: When users look for better performances and are ready to pay (i.e., users with profile no. 2), they are interested in higher throughputs (up to their maximum requirement). However, when they seek to save up money (i.e., users with profile no. 5), they may sacrifice their service quality and look for lower throughputs (until 


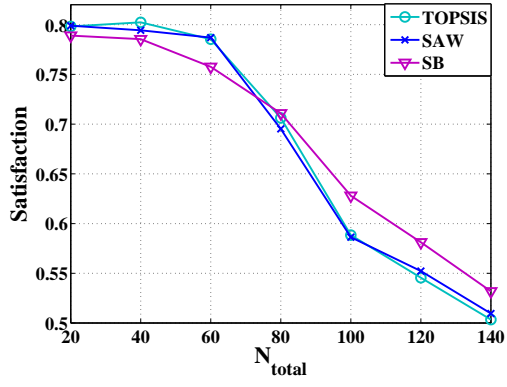

(a) for inelastic sessions

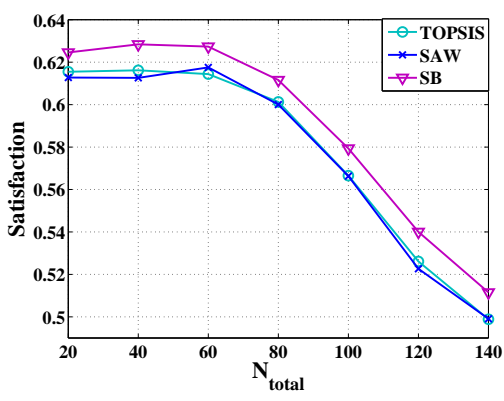

(b) for streaming sessions

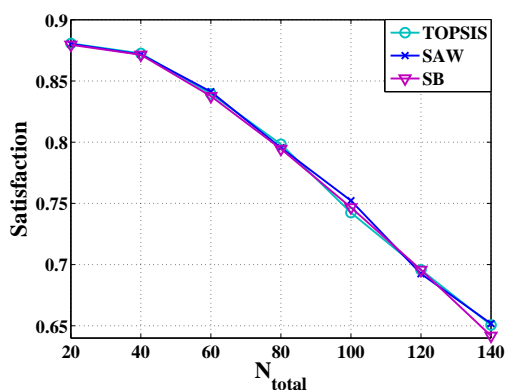

(c) for elastic sessions

Fig. 3. User-perceived satisfaction

their minimum requirement). While session requirements are transparent to SAW and TOPSIS, they are integrated within the QoS satisfaction function when using SB (Eq. 2) : $\alpha$ and $\beta$ determine the shape of the sigmoid function and thus depend on the minimum and the maximum throughput requirements.

When users are ready to pay for better performances, SAW and TOPSIS lead to the Premium service class and SB to the Regular one for $0.5 \mathrm{Mb} / \mathrm{s}$ sessions. SAW and TOPSIS decisions are considered to be oversized; the Regular service class actually provides users with twice their average longterm throughput.

The mean waiting delay and the packet drop probability are respectively depicted in Fig. 4 and 5. Since all methods provide the same QoS level, we deduce that the Premium service class is oversized for $0.5 \mathrm{Mb} / \mathrm{s}$ sessions. In comparison with $\mathrm{SB}$, no performance improvement is observed. Therefore, on average, SB charges less (avoids oversized decisions) and carries out higher user satisfaction (Fig. 3(b)).

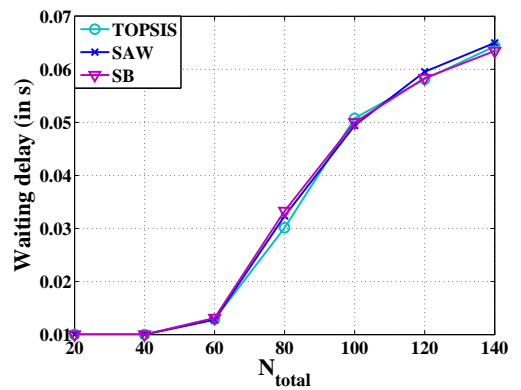

Fig. 4. Mean packet delay for streaming sessions

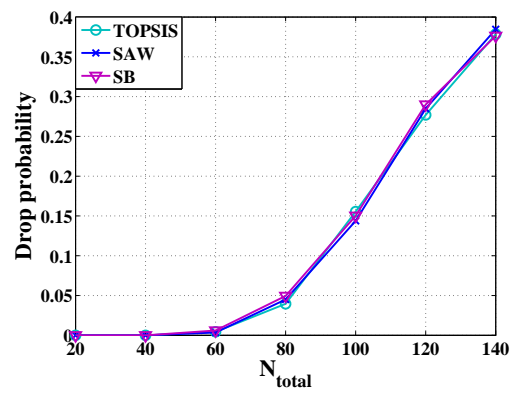

Fig. 5. Packet drop probability for streaming sessions

3) Elastic sessions: Because elastic sessions accommodate with available bandwidth, undersized and oversized decisions do not technically exist. Although theoretically SB may reach different solutions from SAW and TOPSIS (since SB takes into account the comfort throughput), they practically all lead to the same decisions, given our simulation model and parameters. As a consequence, they all ensure the same performances and user satisfaction (Fig. 3(c)).

\section{CONClusion}

In this paper, a hybrid approach for RAT selection is presented. Focusing on the user side, we introduce a satisfactionbased (SB) MCDM method. Since it considers traffic class and needs, SB leads to the most appropriate decisions. Simulation results show that SB brings better performances and charges, on average, less than existing multi-criteria decisionmaking methods, thus providing higher user satisfaction. It actually best meets QoS requirements for the best cost: In comparison with enhanced SAW and TOPSIS methods, when session needs are stringent and inflexible, SB avoids undersized choices and leads to a high enough priority service class. Furthermore, when higher bandwidth guarantees do not improve session performances, SB avoids oversized choices and leads to a low enough priority service class.

\section{REFERENCES}

[1] M. Ibrahim, K. Khawam, and S. Tohme, "Network-Centric Joint Radio Resource Policy in Heterogeneous WiMAX-UMTS Networks for Streaming and Elastic traffic," in Proc. IEEE WCNC, April 2009.

[2] M. C. Lucas-Estañ, J. Gozalvez, and J. Sanchez-Soriano, "Integer Linear Programming Optimization of Joint RRM Policies for Heterogeneous Wireless Systems," Computer Networks Journal, January 2012.

[3] K. Khawam, M. Ibrahim, J. Cohen, S. Lahoud, and S. Tohme, "Individual vs. Global Radio Resource Management in a Hybrid Broadband Network," in Proc. IEEE ICC, June 2011.

[4] D. Niyato and E. Hossain, "Dynamics of Network Selection in Heterogeneous Wireless Networks: An Evolutionary Game Approach,” IEEE Transactions on Vehicular Technology, May 2009.

[5] E. Stevens-Navarro and V. Wong, "Comparison between Vertical Handoff Decision Algorithms for Heterogeneous Wireless Networks," in Proc. IEEE VTC, May 2006.

[6] O. E. Falowo and H. Anthony Chan, "Dynamic RAT Selection for Multiple Calls in Heterogeneous Wireless Networks Using Group DecisionMaking Technique," Computer Networks Journal, March 2012.

[7] M. E. Helou, S. Lahoud, M. Ibrahim, and K. Khawam, "A Hybrid Approach for Radio Access Technology Selection in Heterogeneous Wireless Networks," in Proc. EW, April 2013.

[8] "IEEE Standard for Architectural Building Blocks Enabling NetworkDevice Distributed Decision Making for Optimized Radio Resource Usage in Heterogeneous Wireless Access Networks," IEEE 1900.4-2009. 